\title{
Interpersonal, Affective and Compulsive Features of Driven Exercise in
}

\section{Anorexia Nervosa}

Kolar, D. R. ${ }^{1, \dagger, *}$, Kaurin, A. ${ }^{2, \dagger}$, Meule, A. ${ }^{1,3}$, Schlegl, S. ${ }^{1,3}$, Dittmer, N. ${ }^{3}$, \& Voderholzer, U. ${ }^{1,3,4}$

${ }^{1}$ Department of Psychiatry and Psychotherapy, University Hospital, Ludwig-MaximiliansUniversity Munich, Munich, Germany

${ }^{2}$ Faculty of Health/School of Psychology and Psychiatry, Witten/Herdecke University, Witten, Germany

${ }^{3}$ Schoen Clinic Roseneck, Prien am Chiemsee, Germany

${ }^{4}$ Department of Psychiatry and Psychotherapy, University Medical Center Freiburg, Freiburg, Germany

Please note that this is a pre-print accepted at Journal of Affective Disorders (draft version 14/03/2022). Please cite as follows:

Kolar, D.R., Kaurin, A., Meule, A., Schlegl, S., Dittmer, N. \& Voderholzer, U. (in press). Interpersonal, Affective and Compulsive Features of Driven Exercise in Anorexia Nervosa. Journal of Affective Disorders. https://doi.org/10.1016/j.jad.2022.03.044

${ }^{\dagger}$ shared first authorship

${ }^{*}$ Corresponding author:

LMU University Hospital, Department of Psychiatry and Psychotherapy, Nussbaumstr. 7, 80336 Munich, Germany

Tel.: +49 $894400-52721$ 
Fax.: +49 $894400-54572$

Email: David.kolar@med.uni-muenchen.de

Word count: 5233 words

Abstract word count: 250 words 


\begin{abstract}
Background: This preregistered (https://osf.io/g9ajb) study sought to integrate the current literature on trait compulsivity into maintenance models of driven exercise in anorexia nervosa (AN). We tested whether compulsivity increases the likelihood of driven exercise via interpersonal and affect-regulatory pathways.

Methods: We used multilevel structural equation modeling to test the hypothesis that trait compulsivity predicts a stronger within-person link between affect-regulatory difficulties or interpersonal sensitivity and driven exercise in female adolescents and adults with AN. We used data from five assessments across inpatient treatment and 6-months follow-up of 207 adult and adolescent patients with AN (1036 datapoints).
\end{abstract}

Results: In line with our hypotheses, patients who generally experienced more affectregulatory difficulties or stronger interpersonal sensitivity tended to engage in more driven exercise. Moreover, high levels of trait compulsivity amplified the effect of interpersonal sensitivity on driven exercise across time. Contrary to our hypotheses, the link between affect regulation and driven exercise was not moderated by compulsivity. Similar effects on general ED psychopathology were found, but no cross-level moderation of compulsivity.

Limitations: Due to sample size, potential age- and subtype-dependent effects were not analyzed.

Conclusion: Our results suggest that driven exercise coincides with self-reported experiences of interpersonal sensitivity and that this link varies as a function of compulsivity such that the within-person coupling is stronger among those scoring high on compulsivity. To derive clinically useful functional models of driven exercise, future studies might use intensive longitudinal data to investigate its momentary associations with affect and interpersonal sensitivity in the context of compulsive traits.

Keywords: eating disorders, compulsive exercise, compulsivity, personality disorders 
Interpersonal, Affective and Compulsive Features of Driven Exercise in Anorexia Nervosa

\section{Introduction}

Anorexia nervosa (AN) is a severe eating disorder (ED) that primarily affects women in their adolescence and early adulthood (Nagl et al., 2016; Smink et al., 2012). AN often takes a chronic course (Zipfel et al., 2000) and recovery frequently requires specialized inpatient treatment (Schlegl et al., 2016). Diagnostic features of AN comprise severe weight loss, food restriction and body dissatisfaction. Food restriction is the main driver for severe weight loss, the primary symptom of AN, but a substantial number of individuals with AN further engage in behaviors that impact energy expenditure, such as driven exercise (Dalle Grave et al., 2008; Fietz et al., 2014). Several psychological factors have been suggested to perpetuate driven exercise, including affect-regulatory difficulties, interpersonal problems and individual differences reflective of both, such as pathological personality traits (e.g., compulsivity, impulsivity; Lavender et al., 2017). The present study sought to integrate the current literature on compulsivity into maintenance models of driven exercise in AN. To this end, we tested whether compulsivity increases the amount of driven exercise via interpersonal and affective pathways in an inpatient sample of adolescents and adults with AN.

Depending on sample characteristics and assessment method, approximately $31 \%$ to $80 \%$ of individuals with AN report engaging in a driven exercise routine (Dalle Grave et al., 2008; Fietz et al., 2014). Driven exercise - also referred to as excessive exercise (Davis et al., 1997), exercise dependence (Hausenblas \& Downs, 2002) or compulsive exercise (Dittmer et al., 2018; Meyer et al., 2011) - is described as an urge to exercise or to engage in physical activity to influence body weight or shape, to achieve positive consequences of exercise (e.g., being proud, initiate social contact), or to attenuate momentary negative affect (Kolar \& Gorrell, 2021). ${ }^{1}$ Understanding the role of driven exercise for the etiology and maintenance of AN is key, because it is associated with worse treatment outcome (Dalle Grave et al., 2008; Danielsen et al., 2016; Stiles-Shields et al., 2015). Only recently specific interventions have 
been developed that directly target patterns of driven exercise in AN, with some evidence suggesting good efficacy (Dittmer et al., 2020; Hay et al., 2018).

Affect-regulatory difficulties are considered to represent a key maintenance factor of eating pathology in general and AN specifically (Lavender et al., 2015; Oldershaw et al., 2015; Perthes et al., 2021; Prefit et al., 2019). Recent maintenance models of AN conceptualize disordered eating behaviors as maladaptive attempts to regulate negative affective states (Haynos \& Fruzzetti, 2011). Beyond decreases in negative affect, momentary increases in positive affect following driven exercise have also been observed in adults and adolescents with AN (Engel et al., 2013; Kolar et al., 2020; Reichert et al., 2020; Vansteelandt et al., 2007). Thus, both positive and negative short-term reinforcement are considered to maintain driven exercise, and these short-term effects may explain persistent engagement in driven exercise despite long-term negative and potentially life-threatening consequences (Coniglio, Cooper \& Selby, 2021; Kolar \& Gorrell, 2021).

Besides intrapersonal affect-regulatory difficulties, individuals with EDs also struggle to regulate negative affect in interpersonal situations (Christensen \& Haynos, 2020). Metaanalytic evidence suggests that EDs are associated with atypical processing of stimuli suggestive of negative social evaluation (Monteleone et al., 2018), and perceptions of negative social evaluation (e.g., bullying, teasing, critical social feedback) are associated with increased risk for ED symptoms (Fairweather-Schmidt \& Wade, 2017; Menzel et al., 2010; Puhl et al., 2017). In addition, a recent network analysis further highlighted the importance of interpersonal sensitivity to negative social events in AN (Solmi et al., 2019). It is plausible to assume that interpersonal problems in AN lead to social maladjustment (i.e., social inhibition, poor emotional support from others), which then maintain ED pathology, for example by increased ED behaviors to reduce the assumed likelihood of negative body-related evaluations by others (Arcelus et al., 2013). Relatively little research has been conducted to investigate associations of interpersonal problems with driven exercise. A study found that appearance- 
related social rejection may account for the relationship between social anxiety and driven exercise (Linardon et al., 2017), indicating that driven exercise might serve as a coping mechanism. Higher levels of driven exercise were associated with feeling more distant to siblings, significant others and roommates in an ego-network analysis (a social network that is focused on one actor instead of specific groups or the complete network) of college students (Patterson \& Goodson, 2018). Furthermore, exercising for social reasons (e.g., exercising to meet other people, to not miss out on social life) was correlated with increased driven exercise (MacLaren \& Best, 2007), indicating a potential interpersonal function. Driven exercise may, thus, be a behavior to cope with (perceived) interpersonal problems, especially for individuals that experience high levels of interpersonal sensitivity towards negative social events. Together with the general link of interpersonal problems with disordered eating, these findings suggest that interpersonal sensitivity may play an important role in the maintenance of driven exercise in individuals with AN. However, there is a lack of studies directly investigating this relationship.

Cross-sectional research suggests that dispositional compulsivity might be positively linked to affect-regulatory difficulties and driven exercise (Goodwin et al., 2011). However, studies that integrate findings on pathological personality traits (such as compulsivity) into affective and interpersonal maintenance models of AN are missing. Individuals with AN that also exhibit high dispositional compulsivity showed higher levels of restraint, eating and weight concerns relative to those with low levels of compulsivity (Lavender et al., 2017). Similarly, across EDs, driven exercise is associated with personality characteristics related to compulsivity such as low novelty-seeking and high persistence in EDs (Shroff et al., 2006). Individuals with AN that were further described as overregulated (i.e., high compulsivity and low stimulus seeking) experienced more negative and less positive affect in their daily lives, and exercised more often compared to underregulated (i.e., high stimulus seeking, oppositionality and self-harm) and low personality pathology individuals in an ecological 
momentary assessment (EMA) study (Lavender et al., 2013). An important context within which negative affective states are frequently experienced in individuals scoring high on personality pathology such as compulsivity are interpersonal situations (Wright \& Ringwald, 2022). It is, therefore, plausible to assume that the frequently observed enhanced levels of compulsivity in individuals with AN (Claes et al., 2002; Godier \& Park, 2014; Lavender et al., 2017; Wildes \& Marcus, 2013) might also attenuate the link between interpersonal sensitivity with disordered eating in general and driven exercise in particular. However, studies that integrate findings on pathological personality traits (such as compulsivity) into affective and interpersonal maintenance models of AN are missing.

\subsection{The Current Study}

Compulsivity represents an important individual difference that is linked to the maintenance of $\mathrm{AN}$, as well as affect-regulatory and interpersonal difficulties. Therefore, we investigate whether compulsivity is associated with driven exercise via affect regulatory and interpersonal pathways during and after inpatient treatment of adolescents and adults with full-threshold or atypical AN.

To this end, we preregistered two sets of questions: First, we were interested in investigating potential relations of affect regulation with driven exercise within and across individuals (Aim 1). Specifically, we hypothesized that affect-regulatory difficulties would be linked to driven exercise both at the within and between-person level (H0). We then examined the hypotheses that compulsivity would be positively associated with affect-regulatory difficulties (H1a) and driven exercise (H1b). Second, we evaluated potential effects of compulsivity on within-person links of affect-regulatory difficulties with driven exercise (Aim 2). This second set of hypotheses focused on cross-level moderating effects of compulsivity on the within-person association of these variables across five measurements during and after inpatient treatment, with an amplification of the association of affect-regulatory difficulties and driven exercise by higher levels of compulsivity (H2). In addition, we preregistered 
exploring the same hypotheses regarding interpersonal sensitivity and the moderation of their link to driven exercise by compulsivity. On an exploratory level, we also investigated whether these relations are specific to driven exercise or are applicable to disordered eating in general.

\section{Method}

All study procedures were approved by the Institutional Review Board of Medical Faculty of the Ludwig Maximilian University of Munich (no. 060-13). Participants were drawn from a randomized controlled trial assessing a group intervention integrating exercisebased elements into a cognitive behavioral therapy framework in addition to a specialized ED inpatient treatment (Dittmer et al., 2020). Thus, the present study represents a secondary data analysis. For a detailed description of the study flow, additional assessment procedures, treatment details and trial outcomes not relevant to this study please refer to Dittmer et al. (2020). All analyses were preregistered by the first authors at OSF (https://osf.io/g9ajb). Data and code to reproduce main analyses are available at https://osf.io/ps53y.

\subsection{Participants}

The sample comprised $N=207$ female participants, all receiving inpatient treatment at the Schoen Clinic Roseneck, Germany. $N=120$ had an AN restrictive subtype, $n=35$ had an AN binge-purge subtype (DSM-IV 307.1) and $n=52$ had an atypical AN (DSM-IV 307.50) diagnosis (aged 13 to 44 years, $M=19.2, S D=5.52 ; n=108$ adolescents, $n=99$ adults), and all participants frequently engaged in driven exercise, fulfilling the criteria defined in Dittmer et al. (2018): Prior to admission, (A) all participants excessively exercised in response to obsessions or rigid rules and exercising was aimed at preventing dreaded consequences or reducing distress; (B) the exercising was time-consuming ( $>1 \mathrm{~h} /$ day), interfered with daily routines, social or work functioning or was continued despite injury, illness or lack of enjoyment. Diagnoses were confirmed using the Structured Inventory for Anorexic and Bulimic Eating Disorders (SIAB-EX; Fichter \& Quadflieg, 2001). Exclusion criteria were a $\mathrm{BMI}<13 \mathrm{~kg} / \mathrm{m}^{2}$ prior to the group intervention for medical safety reasons, current substance or 
alcohol abuse, diagnosis of severe psychiatric (i.e., bipolar and psychotic disorders) or neurological disorders (multiple sclerosis), current suicidal ideation, severe somatic complications that did not allow light or moderate supervised exercise, cognitive impairment due to significant underweight that did not permit attending and following 100-min group sessions, and concurrent exposure-based treatment for obsessive-compulsive disorder. The latter was defined as an exclusion criterion because the additional group intervention and standard cognitive behavioral treatment for obsessive-compulsive disorder contained both exposure with response management (for details, please refer to Dittmer et al., 2020).

Demographic information is displayed in results.

\subsection{Procedures}

Within one week after admission to inpatient treatment, all patients were screened for eligibility in the intervention study. If patients were eligible and after informed consent, patients were randomized to either treatment-as-usual (TAU) or routine treatment amended by a four-week group intervention focusing on healthy exercise behaviors (two 100 mins sessions each week). Randomization was conducted at hospital ward-level ( $N=112$ patients receiving TAU and exercise group intervention, $N=95$ patients receiving TAU only). Main outcome of the RCT was that the additional exercise-focused group intervention resulted in a significant reduction in the severity of driven exercise compared to TAU. However, no effects of the additional group intervention on emotion regulation, weight gain, eating disorder and general psychopathology were found (for a thorough description of the results see Dittmer et al., 2020). TAU for all participants consisted of multimodal cognitive behavioral therapy with intensive general psychiatric care. All patients received individual psychotherapy (1-2 times per week), eating disorder and non-specific problem-solving group treatment (up to three times per week), supervised meals and frequent weight monitoring (for further details see Dittmer et al., 2020). Self-report measures were assessed at five time points: admission (T0; within the first week after admission), pre-intervention (T1; within three days before the start 
of the healthy exercise behavior group intervention), post-intervention (T2; within three days after the last intervention session, roughly four weeks after T1), discharge (T3, within one week before discharge) and six months follow-up (T4; see supplement file S2 for assessment plan), resulting in $N=1035$ data points. Due to missing data, only $n=937$ data points were included in the analysis. In the current study, we controlled statistically for any group effects as these were not of primary interest.

\subsection{Measures}

Participants completed the following measures at admission and at following assessments if indicated. Reliability estimates for repeated measures were assessed based on generalizability theory (Cranford et al., 2006; Cronbach et al., 1972). To assess the proportion of total variance accounted for at the between-person level, we calculated intraclass correlations (ICC).

Consequently, 1.0 - ICC expresses the proportion of within-person variance and provides an estimate of the variability of our measures across treatment. Supplement file 1 illustrates longitudinal individual differences in average levels and within-person variability of driven exercise, affect-regulatory difficulties and interpersonal sensitivity at assessments across treatment groups.

Commitment to Exercise Scale (CES). The CES is an 8-item self-report scale that is widely used for the dimensional assessment of driven exercise in EDs (Davis et al., 1993; German version: Zeeck et al., 2017). Two core components of driven exercise, adherence to exercise rules and physical or psychological burden of exercise, are assessed with the measure. In accordance with Thome and Espelage (2007), we applied a four-point scale response format, as this version showed higher reliability. The scale showed good reliability for between-person $\left(\mathrm{R}_{\mathrm{kRn}}=.81\right)$ and within-person variations $\left(\mathrm{R}_{\mathrm{cn}}=.80\right)$ when averaged over time. The CES total variance was explained by both within and between-person variance to a similar degree $(\mathrm{ICC}=.47)$. 


\section{Dimensional Assessment of Personality Pathology - Compulsivity Subscale}

(DAPP-Comp).Compulsivity was assessed at admission with the German version of the DAPP-Comp (Pukrop et al., 2001). The DAPP is a self-report measure comprised of 290 items that assesses personality pathology across 18 traits with a four-factor higher-order structure (emotion dysregulation, dissocial behavior, inhibitedness and compulsivity; Livesley \& Jackson, 2009). The DAPP-Comp consists of 16 items and is the only scale loading on the higher-order factor compulsivity. The scale score showed good reliability in our sample (McDonald's $\omega=.92)$.

Difficulties in Emotion Regulation Scale (DERS). We used the DERS to measure affect-regulatory difficulties (Gratz \& Roemer, 2004). It is a 36-item questionnaire assessing difficulties in emotion regulation across six factors, namely non-acceptance of emotional responses, difficulty engaging in goal directed behavior, impulse control difficulties, lack of emotional awareness and limited access to emotion regulation strategies. The items are rated on a Likert scale ranging from 1 - almost never to 5 - almost always. We used the global mean score as a measure of affect-regulatory difficulties at all measurement time points. The reliability of the German DERS (Ehring et al., 2010) was high at both the between-person $\left(\mathrm{R}_{\mathrm{kRn}}=.90\right)$ and within-person level $\left(\mathrm{R}_{\mathrm{cn}}=.87\right)$ when averaged over time. A moderate degree of the DERS total variance was explained by between-person variance $(\mathrm{ICC}=.62)$.

Eating Disorder Inventory 2 - Social Insecurity (EDI-SI). We used mean scores of the social insecurity subscale of the Eating Disorder Inventory-2 (Garner, 1991) to assess interpersonal sensitivity, that is how sensitive or insecure a person feels when facing (perceived) social threats. The EDI-SI is comprised of eight items measuring for example how tense one feels in social groups on a scale ranging from 1 - never to 6 - always. Different to the English version, the German translation maintains the item scale for scoring, instead of combining the lower three response categories into zero and counting the upper three categories from one to three. The scale was repeatedly administered at every measurement 
timepoint. The reliability of the German EDI-SI scale (Thiel et al., 1997) was high at the between-person level $\left(\mathrm{R}_{\mathrm{kRn}}=.91\right)$, but low for within-person variation $\left(\mathrm{R}_{\mathrm{cn}}=.32\right)$ when averaged over time. Between-person variance explained a moderate proportion of the EDI-SI total variance $(\mathrm{ICC}=.66)$.

Eating Disorder Examination Questionnaire (EDE-Q). Disordered eating was measured at every assessment using the EDE-Q (Fairburn \& Beglin, 1994). The EDE-Q assesses eating disorder psychopathology such as eating restraint, fear of loss of control over eating, and dissatisfaction with body or weight. The 7-item scale reflects on how many days an item was present within the last 28 days ranging from 0 - not at all to 7 - every day. Four subscales describing distinct features of disordered eating can be computed: eating restraint, eating, weight, and shape concern. In our sample, the German version of the EDE-Q (Hilbert et al., 2007) showed good between-person $\left(\mathrm{R}_{\mathrm{kRn}}=.79\right)$ and within-person reliability $\left(\mathrm{R}_{\mathrm{cn}}\right.$ $=.89$ ) averaged over time. $\mathrm{ICC}=.39$ indicates substantial within-person variability across assessments. The EDE-Q global scale was used as an outcome measure in a supplementary analysis.

\subsection{Data Analysis}

Recurrently assessing affect-regulatory difficulties, interpersonal sensitivity and driven exercise results in a hierarchical data structure, where subjective ratings (within-person level) are nested within individuals (between-person level). Separating the variance into each level returns information about the proportion of subjective ratings that is attributable to individual differences as opposed to fluctuations in subjective ratings across treatment/assessment points.

We used multilevel structural equation modeling (MSEM; Kaurin et al., 2020; Sadikaj et al., 2021), to test whether affect regulation and driven exercise were linked to each other across time, and whether that covariation pattern was more pronounced among those who indicated higher compulsivity scores. MSEM is a modern analytical approach that merges 
traditional structural equation modeling (e.g., multiple outcome variables analyzed simultaneously) with multilevel modeling (e.g., analyzing hierarchical data). Thus, MSEM can accommodate repeated assessments nested within persons and allows for decomposing the total variance into the latent Level 2 (between-person) variance and the Level 1 (withinperson) residual variance. It also allows for the estimation of random effects (i.e., intercepts and slopes that vary across individuals). All models were estimated in Mplus with Bayesian parameter estimation (version 8.4; Muthén \& Muthén, 2019), and missing data were assumed to be missing at random. A Bayesian approach to MSEM uses all available data in estimation: with increasingly large samples, it provides similar results to Full Information Maximum Likelihood to address missing data (Asparouhov \& Muthén, 2010).

To validate our Aim 1 models and as a test of respective hypotheses, we regressed ratings of driven exercise on ratings of affect-regulatory difficulties at Level 1 (within-person) and tested their correlation at Level 2 (between-person; H0). To test hypothesis 1, we then examined whether dispositional compulsivity was related to individual differences in random intercepts of affect-regulatory difficulties (H1a) and driven exercise (H1b). For our Aim 2 analyses, we examined whether dispositional compulsivity moderated the within-person link between affect-regulatory difficulties and driven exercise (i.e., cross-level interaction; H2). Figure 1 depicts the path diagram for the Aim 2 Model. Because our sample comprised a broad age span (i.e., female adolescents and adults) and because participants were randomized to TAU or TAU amended with an additional exercise group treatment, we included age as well as treatment group as covariates at the between-person level. That is, to statistically control for the effects of each, random slopes and intercepts were regressed on these covariates at Level 2. To account for intraindividual effects of weight gain and treatment stage, driven exercise was regressed on assessment time point and body weight at the withinperson level (Level 1). This set of analyses (i.e., H0-H2) was then repeated with interpersonal 
sensitivity as the independent variable to explore whether the within-person coupling between interpersonal sensitivity and driven exercise was also amplified by compulsivity.

\section{Results}

\subsection{Sample Characteristics}

Table 1 provides an overview of sample characteristics at admission and discharge. Attrition rates from admission to follow-up were acceptable, with no less than $n=151$ ratings at any assessment (72.9\%, for EDI-SI at follow-up). Within-person and between-person correlations of key measures are displayed in Table 2. Except for BMI and age, significant correlations were found between all measures. Our participants reported significantly higher levels of driven exercise at admission $(M=2.93, S D=0.68)$ compared to non-clinical populations $(M=2.02, S D=0.63 ; t(804)=-17.55, p<.001, d=1.39)$. Compulsivity, driven exercise, social insecurity, or affect-regulatory difficulties at admission did not vary as a function of AN subtype (all $p>.521)$.

\subsection{Affect-regulatory Difficulties, Compulsivity and Driven Exercise}

Aim 1 Model. We first tested the hypothesis that affect-regulatory difficulties were positively linked to dimensional levels of driven exercise at the within- and between-person levels. We found a positive significant link at the within-person level, indicating that on a given assessment occurrence, when individuals reported more difficulties in affect regulation, they also tended to report enhanced levels of driven exercise. A similar, albeit slightly less pronounced association emerged at the between-person level, suggesting that individuals, who - on average - tended to experience more affect-regulatory difficulties, also tended to engage more frequently in driven exercise ( $\mathrm{H} 0$, see Aim 1 column of Table 3A for parameter estimates and confidence intervals). Within-person effects for assessment time point and body weight emerged, as well as a between-person effect of age (see supplement file 3).

Aim 2 Model. Turning to H1a/b, we found that both, affect-regulatory difficulties $(\mathrm{H} 1 \mathrm{a} ; \beta=.19, \mathrm{CI}: .04 ; .32$; Table $3 \mathrm{~A})$ and driven exercise $(\mathrm{H} 1 \mathrm{~b} ; \beta=.22$, CI: .07; .36; Table 
3A), were linked to higher average levels of dispositional compulsivity. In a next step, we examined whether compulsivity was a significant cross-level moderator of the Level 1 withinperson link established in the Aim 1 model. In contrast to our hypotheses, no significant cross-level interaction effect emerged, suggesting that the within-person coupling between affect-regulatory difficulties and driven exercise did not vary as a function of compulsivity (H2, see final row of Table 3A, "compulsivity $\rightarrow$ random slope"). Of note, a cross-level effect emerged for treatment allocation, suggesting that in the intervention group, the positive link between affect-regulatory difficulties and driven exercise was more strongly pronounced.

\subsection{Interpersonal Sensitivity, Compulsivity, and Driven Exercise}

We also explored whether temporal links between interpersonal sensitivity and driven exercise varied as a function of dispositional compulsivity, testing models analogous to those described above, but using EDI-SI as an indicator for interpersonal sensitivity. Of note, significant within-person effects for time and weight and a between-person effect for age as a covariate were found in both models (supplement file 3).

Aim 1 Model. Self-rated interpersonal sensitivity was positively linked to driven exercise at the within- and between-person level (Table 3B).

Aim 2 Model. Higher levels of interpersonal sensitivity as well as higher levels of driven exercise (both $\beta=.21$, CI: .06; .36; Table 3B) were linked to higher average levels of dispositional compulsivity. A significant cross-level interaction effect emerged, wherein compulsivity moderated the positive within-person link between interpersonal sensitivity and driven exercise (see final row of Table 3B, “compulsivity $\rightarrow$ random slope"). More specifically, we found that this positive within-person link was significantly elevated at mean levels of compulsivity $(\beta=0.31,95 \%$ CI $[0.22, .40])$ as well as one SD above the mean $(\beta=0.70,95 \% \mathrm{CI}[0.45,0.93])$, but not at compulsivity scores at one SD below the mean $(\beta=-0.08,95 \% \mathrm{CI}[-0.36,0.19])$. Thus, for patients with AN and high levels of compulsivity, interpersonal sensitivity was more strongly linked to driven exercise relative to those 
characterized by low levels of dispositional compulsivity. An illustration of significant interaction effects is provided in Figure 2.

\subsection{Additional Exploratory Analyses}

We also explored whether associations of compulsivity, affect-regulatory difficulties, and interpersonal sensitivity were specific to driven exercise or whether they generalized to symptoms of disordered eating more broadly. To test this possibility, we fitted models analogous to those described above using the EDE-Q global scores as our outcome measure and an indicator of core ED psychopathology. Overall, similar patterns of results emerged regarding within- and between-person associations of compulsivity, affect-regulatory difficulties, and personal sensitivity. However, we found no cross-level interaction effect of compulsivity on the within-person link of either affect-regulatory difficulties or interpersonal sensitivity with general ED psychopathology (see supplementary Table 4).

\section{Discussion}

Previous cross-sectional research suggested that the link between compulsivity and driven exercise might be partially explained by affect-regulatory difficulties in individuals with a diagnosis of AN.

In this study, we investigated these links in a mixed adolescent and adult sample of women with AN to test how the link between affect regulation and driven exercise unfolded within individuals throughout inpatient treatment and a follow-up period. As expected, a significant positive within-person association of affect-regulatory difficulties with driven exercise emerged (H0). At the between-person level we found that women with AN, who, on average, experienced more affect-regulatory difficulties, were also more likely to report high levels of driven exercise (H0), and that compulsivity was positively related to driven exercise (H1b). Similarly, women who, on average, reported higher levels of compulsivity also reported more affect-regulatory difficulties (H1a). Regarding the central hypothesis of this study (H2), we found no evidence that the within-link between affect-regulatory difficulties 
and driven exercise varied as a function of dispositional compulsivity. In other words, in our sample compulsivity did not amplify the link between affect-regulatory difficulties and driven exercise. At the same time, women with a diagnosis of AN, who - on average - described themselves as highly interpersonally sensitive, tended to engage more frequently in driven exercise. Dispositional compulsivity was further positively related to higher average levels of interpersonal sensitivity, and enhanced the positive association between interpersonal sensitivity and driven exercise.

A similar pattern of results emerged for models including ED pathology as dependent variable. However, a significant cross-level moderation of compulsivity did not emerge in these analyses. Thus, dispositional compulsivity did not account for the temporal coupling of affect-regulatory difficulties or interpersonal sensitivity on disordered eating. An explanation could be that healthy exercising is often a social activity (e.g., as an accepted form of meeting new people, MacLaren \& Best, 2007), and thus this normative aspect of social function remains in driven exercise. Thus, patients with $\mathrm{AN}$ and high compulsivity might more rigidly engage in driven exercise when experiencing high interpersonal sensitivity due to the perceived social functionality of exercise, whereas women describing themselves as less compulsive might be more likely to explore other regulative behaviors.

Current maintenance models of AN particularly focus on affect-regulatory difficulties. In line with previous work (Monteleone et al., 2018; Wang et al., 2022), our results extend this view and suggest that interpersonal sensitivity may play another important role in maintaining AN symptoms over time. To better unterstand how these processes interplay in the daily lives of individuals, and thus aid the development of clinically useful interventions, future research might investigate dynamic links of interpersonal sensitivity and driven exercise via EMA. This would allow to disentangle short-term temporal dynamics and highlight tangible social functions of driven exercise, which might be positively (increasing positive interpersonal interactions) or negatively (avoiding conflict) reinforcing. In addition, 
experimental studies investigating whether social stressors might impact duration, intensity or exercise satiation (i.e., achieving a state of satisfaction or sense of completion when exercising; see Barker, Kolar, Lazzer \& Keel, 2021) of driven exercise episodes could provide additional insight into potential reinforcing mechanisms. Finally, because we only assessed a specific component of perceived social threats than actual social stressors, future studies might further elaborate a more fine-grained understanding of the routes via which social stressors impact driven exercise in AN. One possibility going forward would be to differentiate the impact of diverse social contexts (e.g., schools, family, peers).

Our findings are also of importance for clinical practice. Directly targeting compulsivity might be promising to enhance treatment effects on driven exercise for individuals with high levels of compulsivity. Similarly, focusing on interpersonal sensitivity, for example by explicitly addressing social fears in exposure therapy during inpatient treatment, might be further helpful in the treatment of AN.

\subsection{Limitations}

This study is a secondary analysis of a randomized controlled trial dataset (Dittmer et al., 2020) that tested a group intervention integrating exercise-based elements into a cognitive-behavioral framework. Although our analyses were controlled for differences between intervention groups, the setting of the study might have influenced our findings. For example, it is conceivable that temporal associations of affective and interpersonal processes with driven exercise are more strongly moderated by compulsivity outside of inpatient treatment, because the rather strict setting of inpatient treatment might have prevented participants from engaging in driven exercise as they would have in daily life.

Notwithstanding, our participants exhibited substantially high levels of driven exercise. The EDI-SI scale used to assess interpersonal sensitivity showed low within-person reliability, why we might have underestimated the association of driven exercise and interpersonal sensitivity within individuals. However, some authors argue for more relaxed criteria of 
within-person reliability, especially when effects are also measured at the between-person level as in our study (Nezlek, 2016). In addition, our analysis was based on a self-report measure of driven exercise and future studies might want to use more objective measures of exercise such as actigraphy. Our study is characterized by larger intervals, which might lack precision to capture fine-grained temporal processes. Future research might use EMA, which allows studying short-term temporal within-person links (e.g., on an hourly or daily level). This could facilitate detecting further affect regulation and contextual features that are functionally related to driven exercise and investigating their moderation by compulsivity. Our sample size was sufficient at both levels for MSEM, but rather small compared to other studies using similar analysis strategies (e.g., Kaurin et al., 2020), as sample size calculation was based on power for RCT outcomes. Thus, our study likely lacks power to detect crosslevel moderation effects of compulsivity on the link between affect regulation and driven exercise, although we used a Bayesian approach for obtaining more reliable estimates. Another limitation is that we did not compare AN subtypes or adolescents with adults, although we controlled for age in general. Given that binge-purge AN is more strongly linked to affect-regulatory difficulties compared to restrictive AN (e.g., Perthes et al., 2021), it would be interesting to check whether these subtypes also differ with regard to within-person associations of affect regulation and interpersonal sensitivity with driven exercise.

\subsection{Conclusion}

Our research contributes to an established body of evidence that emphasizes the significance of affect-regulatory difficulties for driven exercise in $\mathrm{AN}$, and also extends this line of research by suggesting that interpersonal sensitivity might have a similar maintenance function for driven exercise. To enhance the clinical utility and generalizability of our study, future research should corroborate our findings outside of inpatient treatment in daily life. 


\section{Acknowledgments}

We are grateful to the participants who took part in this study, as well as to the staff and research assistants at the Schoen Clinic Roseneck who helped with data collection.

\section{$6 \quad$ Funding}

This study was funded by a research grant from the Swiss Anorexia Nervosa Foundation (Project no. 26-13). The sponsors of the study had no role in study design, data collection, data analysis, data interpretation, or writing of the report.

\section{Author Contributions}

David R Kolar: Conceptualization, Methodology, Data Curation, Writing - Original draft preparation, Visualization. Aleksandra Kaurin: Conceptualization, Methodology, Data Analysis, Writing - Review \& Editing, Visualization. Adrian Meule: Data Curation, Writing - Review \& Editing. Nina Dittmer: Investigation, Resources, Data Curation, Writing Review \& Editing. Sandra Schlegl: Investigation, Resources, Data Curation, Writing Review \& Editing, Project administration, Funding acquisition. Ulrich Voderholzer: Resources, Writing - Review \& Editing, Project administration, Funding acquisition.

\section{Disclosure of conflicts}

The authors declared no conflicts of interest. 


\section{Footnotes}

${ }^{1}$ We use the term driven exercise throughout this manuscript instead of compulsive exercise for two reasons: First, to avoid confusion with dispositional compulsivity. Compulsivity is a broader personality trait not necessarily linked to specific behaviors, and often related to other dispositional traits such as perfectionism, rigidity, inflexibility and need for control. Second, compulsive exercise has recently been suggested as a more categorical and clinical conceptualization that includes explicit reasons for and consequences of exercise that we did not assess on a longitudinal basis (e.g., obsessions, rigid rules based on distorted beliefs about exercise or lack of enjoyment, see Dittmer et al., 2018). 


\section{References}

Arcelus, J., Haslam, M., Farrow, C., \& Meyer, C. (2013). The role of interpersonal functioning in the maintenance of eating psychopathology: a systematic review and testable model. Clin Psychol Rev, 33(1), 156-167. https://doi.org/10.1016/j.cpr.2012.10.009

Asparouhov, T., \& Muthén, B. (2010). Bayesian analysis of latent variable models using Mplus.

Barker, J. L., Kolar, D., Lazzer, A. S.-D., \& Keel, P. K. (2021). Exercise Satiation: A novel theoretical conceptualization for problematic exercise observed in eating disorders. International Journal of Eating Disorders, 1-4. https://doi.org/10.1002/eat.23635

Christensen, K. A., \& Haynos, A. F. (2020). A theoretical review of interpersonal emotion regulation in eating disorders: enhancing knowledge by bridging interpersonal and affective dysfunction. Journal of eating disorders, 8 , 21. https://doi.org/10.1186/s40337020-00298-0

Claes, L., Vandereycken, W., \& Vertommen, H. (2002). Impulsive and compulsive traits in eating disordered patients compared with controls. Personality and Individual Differences, 32(4), 707-714.

Coniglio, K. A., Cooper, M., \& Selby, E. A. (2021). Behavioral reinforcement of pathological exercise in anorexia nervosa. International Journal of Eating Disorders, 1-9. https://doi.org/10.1002/eat.23626

Cranford, J. A., Shrout, P. E., Iida, M., Rafaeli, E., Yip, T., \& Bolger, N. (2006). A procedure for evaluating sensitivity to within-person change: can mood measures in diary studies detect change reliably? Personality \& social psychology bulletin, 32(7), 917-929. https://doi.org/10.1177/0146167206287721

Cronbach, L. J., Gleser, G. C., Nanda, H., \& Rajaratnam, N. (1972). The Dependability of Behavioral Measurements. Theory of Generalizability for Scores and Profiles. John Wiley.

Dalle Grave, R., Calugi, S., \& Marchesini, G. (2008). Compulsive exercise to control shape or weight in eating disorders: prevalence, associated features, and treatment outcome. Compr Psychiatry, 49(4), 346-352. https://doi.org/10.1016/j.comppsych.2007.12.007

Danielsen, M., Ro, O., Romild, U., \& Bjornelv, S. (2016). Impact of female adult eating disorder inpatients' attitudes to compulsive exercise on outcome at discharge and follow-up. $J$ Eat Disord, 4(1), 7. https://doi.org/10.1186/s40337-016-0096-0

Davis, C., Brewer, H., \& Ratusny, D. (1993). Behavioral frequency and psychological commitment: necessary concepts in the study of excessive exercising. J Behav Med, 16(6), 611-628. https://doi.org/10.1007/BF00844722

Davis, C., Katzman, D. K., Kaptein, S., Kirsh, C., Brewer, H., Kalmbach, K., Olmsted, M. P., Woodside, D. B., \& Kaplan, A. S. (1997). The prevalence of high-level exercise in the eating disorders: etiological implications. Compr Psychiatry, 38(6), 321-326. https://doi.org/10.1016/s0010-440x(97)90927-5

Dittmer, N., Jacobi, C., \& Voderholzer, U. (2018). Compulsive exercise in eating disorders: proposal for a definition and a clinical assessment. J Eat Disord, 6, 42. https://doi.org/10.1186/s40337-018-0219-x

Dittmer, N., Voderholzer, U., Monch, C., Cuntz, U., Jacobi, C., \& Schlegl, S. (2020). Efficacy of a Specialized Group Intervention for Compulsive Exercise in Inpatients with Anorexia Nervosa: A Randomized Controlled Trial. Psychother Psychosom, 89(3), 161-173. https://doi.org/10.1159/000504583 
Ehring, T., Tuschen-Caffier, B., Schnülle, J., Fischer, S., \& Gross, J. J. (2010). Emotion regulation and vulnerability to depression: spontaneous versus instructed use of emotion suppression and reappraisal. Emotion, 10(4), 563.

Engel, S. G., Wonderlich, S. A., Crosby, R. D., Mitchell, J. E., Crow, S., Peterson, C. B., Le Grange, D., Simonich, H. K., Cao, L., Lavender, J. M., \& Gordon, K. H. (2013). The role of affect in the maintenance of anorexia nervosa: evidence from a naturalistic assessment of momentary behaviors and emotion. J Abnorm Psychol, 122(3), 709-719. https://doi.org/10.1037/a0034010

Fairburn, C. G., \& Beglin, S. J. (1994). Assessment of eating disorders: interview or self-report questionnaire? Int J Eat Disord, 16(4), 363-370. https://doi.org/doi:10.1002/1098108X(199412)16:4<363::AID-EAT2260160405>3.0.CO;2-\#

Fairweather-Schmidt, A. K., \& Wade, T. D. (2017). Weight-related peer-teasing moderates genetic and environmental risk and disordered eating: twin study. Br J Psychiatry, 210(5), 350-355. https://doi.org/10.1192/bjp.bp.116.184648

Fichter, M. M., \& Quadflieg, N. (2001). Das Strukturierte Interview fuer Anorektische und Bulimische Ess-Stoerungen nach DSM-IV und ICD-10 zur Expertenbeurteilung (SIABEX) und dazugehoeriger Fragebogen zur Selbsteinschaetzung (SIAB-S). Verhaltenstherapie, 11(4), 314-325.

Fietz, M., Touyz, S., \& Hay, P. (2014). A risk profile of compulsive exercise in adolescents with an eating disorder: a systematic review. Advances in Eating Disorders, 2(3), 241-263. https://doi.org/10.1080/21662630.2014.894470

Garner, D. M. (1991). Eating disorder inventory-2. psychological assessment resources Odessa, FL.

Godier, L. R., \& Park, R. J. (2014). Compulsivity in anorexia nervosa: a transdiagnostic concept. Front Psychol, 5, 778. https://doi.org/10.3389/fpsyg.2014.00778

Goodwin, H., Haycraft, E., Willis, A. M., \& Meyer, C. (2011). Compulsive exercise: the role of personality, psychological morbidity, and disordered eating. Int J Eat Disord, 44(7), 655660. https://doi.org/10.1002/eat.20902

Gratz, K. L., \& Roemer, L. (2004). Multidimensional assessment of emotion regulation and dysregulation: Development, factor structure, and initial validation of the difficulties in emotion regulation scale. Journal of psychopathology and behavioral assessment, 26(1), 41-54. https://doi.org/10.1023\%2FB\%3AJOBA.0000007455.08539.94

Hausenblas, H. A., \& Downs, D. S. (2002). How Much is Too Much? The Development and Validation of the Exercise Dependence Scale. Psychology \& health, 17(4), 387-404. https://doi.org/10.1080/0887044022000004894

Hay, P., Touyz, S., Arcelus, J., Pike, K., Attia, E., Crosby, R. D., Madden, S., Wales, J., La Puma, M., Heriseanu, A. I., Young, S., \& Meyer, C. (2018). A randomized controlled trial of the compuLsive Exercise Activity TheraPy (LEAP): A new approach to compulsive exercise in anorexia nervosa. Int J Eat Disord, 51(8), 999-1004. https://doi.org/10.1002/eat.22920

Haynos, A. F., \& Fruzzetti, A. E. (2011). Anorexia Nervosa as a Disorder of Emotion Dysregulation: Evidence and Treatment Implications. Clinical Psychology: Science and Practice, 18(3), 183-202.

Hilbert, A., Tuschen-Caffier, B., Karwautz, A., Niederhofer, H., \& Munsch, S. (2007). Eating Disorder Examination-Questionnaire. Diagnostica, 53(3), 144-154. https://doi.org/10.1026/0012-1924.53.3.144 
Kaurin, A., Dombrovski, A. Y., Hallquist, M. N., \& Wright, A. G. C. (2020). Momentary interpersonal processes of suicidal surges in borderline personality disorder. Psychol Med, 1-11. https://doi.org/10.1017/S0033291720004791

Kolar, D. R., \& Gorrell, S. (2021). A call to experimentally study acute affect-regulation mechanisms specific to driven exercise in eating disorders. Int J Eat Disord, 54(3), 280286. https://doi.org/10.1002/eat.23427

Kolar, D. R., Neumayr, C., Roth, M., Voderholzer, U., Perthes, K., \& Schlegl, S. (2020). Testing an emotion regulation model of physical activity in adolescents with anorexia nervosa: A pilot ecological momentary assessment. Eur Eat Disord Rev, 28(2), 170-183. https://doi.org/10.1002/erv.2706

Lavender, J. M., Goodman, E. L., Culbert, K. M., Wonderlich, S. A., Crosby, R. D., Engel, S. G., Mitchell, J. E., Le Grange, D., Crow, S. J., \& Peterson, C. B. (2017). Facets of Impulsivity and Compulsivity in Women with Anorexia Nervosa. Eur Eat Disord Rev, 25(4), 309-313. https://doi.org/10.1002/erv.2516

Lavender, J. M., Wonderlich, S. A., Crosby, R. D., Engel, S. G., Mitchell, J. E., Crow, S. J., Peterson, C. B., \& Le Grange, D. (2013). Personality-based subtypes of anorexia nervosa: examining validity and utility using baseline clinical variables and ecological momentary assessment. Behav Res Ther, 51(8), 512-517. https://doi.org/10.1016/j.brat.2013.05.007

Lavender, J. M., Wonderlich, S. A., Engel, S. G., Gordon, K. H., Kaye, W. H., \& Mitchell, J. E. (2015). Dimensions of emotion dysregulation in anorexia nervosa and bulimia nervosa: A conceptual review of the empirical literature. Clin Psychol Rev, 40, 111-122. https://doi.org/10.1016/j.cpr.2015.05.010

Linardon, J., Braithwaite, R., Cousins, R., \& Brennan, L. (2017). Appearance-based rejection sensitivity as a mediator of the relationship between symptoms of social anxiety and disordered eating cognitions and behaviors. Eat Behav, 27, 27-32. https://doi.org/10.1016/j.eatbeh.2017.10.003

Livesley, W. J., \& Jackson, D. N. (2009). Manual for the Dimensional Assessment of Personality Pathology-Basic Questionnaire. Sigma Press.

MacLaren, V. V., \& Best, L. A. (2007). Symptoms of Exercise Dependence and Physical Activity in Students. Perceptual and Motor Skills, 105(3_suppl), 1257-1264. https://doi.org/10.2466/pms.105.4.1257-1264

Menzel, J. E., Schaefer, L. M., Burke, N. L., Mayhew, L. L., Brannick, M. T., \& Thompson, J. K. (2010). Appearance-related teasing, body dissatisfaction, and disordered eating: A metaanalysis. Body image, 7(4), 261-270. https://doi.org/10.1016/j.bodyim.2010.05.004

Meyer, C., Taranis, L., Goodwin, H., \& Haycraft, E. (2011, May-Jun). Compulsive exercise and eating disorders. Eur Eat Disord Rev, 19(3), 174-189. https://doi.org/10.1002/erv.1122

Monteleone, A. M., Treasure, J., Kan, C., \& Cardi, V. (2018). Reactivity to interpersonal stress in patients with eating disorders: A systematic review and meta-analysis of studies using an experimental paradigm. Neurosci Biobehav Rev, 87, 133-150.

https://doi.org/10.1016/j.neubiorev.2018.02.002

Muthén, L. K., \& Muthén, B. O. (2019). Mplus User's Guide (Eight Edition ed.). Muthén \& Muthén.

Nagl, M., Jacobi, C., Paul, M., Beesdo-Baum, K., Hofler, M., Lieb, R., \& Wittchen, H. U. (2016). Prevalence, incidence, and natural course of anorexia and bulimia nervosa among adolescents and young adults [journal article]. Eur Child Adolesc Psychiatry, 25(8), $903-$ 918. https://doi.org/10.1007/s00787-015-0808-z 
Nezlek, J. (2016). A Practical Guide to Understanding Reliability in Studies of Within-Person Variability. Journal of Research in Personality, 69. https://doi.org/10.1016/j.jrp.2016.06.020

Oldershaw, A., Lavender, T., Sallis, H., Stahl, D., \& Schmidt, U. (2015). Emotion generation and regulation in anorexia nervosa: a systematic review and meta-analysis of self-report data. Clin Psychol Rev, 39, 83-95. https://doi.org/10.1016/j.cpr.2015.04.005

Patterson, M. S., \& Goodson, P. (2018). The Role of Ego Networks in Compulsive Exercise Behavior Among a Sample of College Sorority Women. J Phys Act Health, 15(10), 755762. https://doi.org/10.1123/jpah.2017-0570

Perthes, K., Kirschbaum-Lesch, I., Legenbauer, T., Holtmann, M., Hammerle, F., \& Kolar, D. R. (2021). Emotion Regulation In Adolescents With Anorexia And Bulimia Nervosa: Differential Use Of Adaptive And Maladaptive Strategies Compared To Healthy Adolescents. International Journal of Eating Disorders, 1-7. https://doi.org/10.1002/eat.23608

Prefit, A. B., Candea, D. M., \& Szentagotai-Tatar, A. (2019). Emotion regulation across eating pathology: A meta-analysis. Appetite, 143, 104438. https://doi.org/10.1016/j.appet.2019.104438

Puhl, R. M., Wall, M. M., Chen, C., Bryn Austin, S., Eisenberg, M. E., \& Neumark-Sztainer, D. (2017). Experiences of weight teasing in adolescence and weight-related outcomes in adulthood: A 15-year longitudinal study. Prev Med, 100, 173-179. https://doi.org/10.1016/j.ypmed.2017.04.023

Pukrop, R., Gentil, I., Steinbring, I., \& Steinmeyer, E. (2001). Factorial structure of the German version of the dimensional assessment of personality pathology-basic questionnaire in clinical and nonclinical samples. J Pers Disord, 15(5), 450-456. https://doi.org/10.1521/pedi.15.5.450.19195

Reichert, M., Schlegel, S., Jagau, F., Timm, I., Wieland, L., Ebner-Priemer, U. W., Hartmann, A., $\&$ Zeeck, A. (2020). Mood and Dysfunctional Cognitions Constitute Within-Subject Antecedents and Consequences of Exercise in Eating Disorders. Psychother Psychosom, 89(2), 119-121. https://doi.org/10.1159/000504061

Sadikaj, G., Wright, A. G. C., Dunkley, D. M., Zuroff, D. C., \& Moskowitz, D. S. (2021). Multilevel structural equation modeling for intensive longitudinal data: A practical guide for personality researchers. In J. F. Rauthmann (Ed.), The Handbook of Personality Dynamics and Processes (pp. 855-885). Academic Press. https://doi.org/10.1016/b978-012-813995-0.00033-9

Schlegl, S., Diedrich, A., Neumayr, C., Fumi, M., Naab, S., \& Voderholzer, U. (2016). Inpatient Treatment for Adolescents with Anorexia Nervosa: Clinical Significance and Predictors of Treatment Outcome. Eur Eat Disord Rev, 24(3), 214-222. https://doi.org/10.1002/erv.2416

Shroff, H., Reba, L., Thornton, L. M., Tozzi, F., Klump, K. L., Berrettini, W. H., Brandt, H., Crawford, S., Crow, S., Fichter, M. M., Goldman, D., Halmi, K. A., Johnson, C., Kaplan, A. S., Keel, P., LaVia, M., Mitchell, J., Rotondo, A., Strober, M., Treasure, J., Woodside, D. B., Kaye, W. H., \& Bulik, C. M. (2006). Features associated with excessive exercise in women with eating disorders. Int J Eat Disord, 39(6), 454-461.

https://doi.org/10.1002/eat.20247 
Smink, F. R., van Hoeken, D., \& Hoek, H. W. (2012). Epidemiology of eating disorders: incidence, prevalence and mortality rates. Curr Psychiatry Rep, 14(4), 406-414. https://doi.org/10.1007/s11920-012-0282-y

Solmi, M, Collantoni, E, Meneguzzo, P, Tenconi, E, Favaro, A. (2019). Network analysis of specific psychopathology and psychiatric symptoms in patients with anorexia nervosa. Eur Eat Disorders Rev, 27, 24- 33. https://doi.org/10.1002/erv.2633

Stiles-Shields, C., DclinPsy, B. B., Lock, J., \& Le Grange, D. (2015). The effect of driven exercise on treatment outcomes for adolescents with anorexia and bulimia nervosa. Int $J$ Eat Disord, 48(4), 392-396. https://doi.org/10.1002/eat.22281

Thiel, A., Jacobi, C., Horstmann, S., Paul, T., Nutzinger, D. O., \& Schüßler, G. (1997). Eine deutschsprachige Version des Eating Disorder Inventory EDI-2. PPmP: Psychotherapie Psychosomatik Medizinische Psychologie.

Thome, J. L., \& Espelage, D. L. (2007). Obligatory exercise and eating pathology in college females: replication and development of a structural model. Eat Behav, 8(3), 334-349. https://doi.org/10.1016/j.eatbeh.2006.11.009

Vansteelandt, K., Rijmen, F., Pieters, G., Probst, M., \& Vanderlinden, J. (2007). Drive for thinness, affect regulation and physical activity in eating disorders: a daily life study. Behav Res Ther, 45(8), 1717-1734. https://doi.org/10.1016/j.brat.2006.12.005

Wang, S. B., Fox, K. R., Boccagno, C., Hooley, J. M., Mair, P., Nock, M. K., \& Haynos, A. F. (2021). Functional assessment of restrictive eating: A three-study clinically heterogeneous and transdiagnostic investigation. Journal of Abnormal Psychology, 130(7), 761-774. https://doi.org/10.1037/abn0000700

Wildes, J. E., \& Marcus, M. D. (2013). Alternative methods of classifying eating disorders: models incorporating comorbid psychopathology and associated features. Clin Psychol Rev, 33(3), 383-394. https://doi.org/10.1016/j.cpr.2013.01.006

Wright, A. G., \& Ringwald, W. R. (2021). Personality Disorders are Dead, Long Live the Interpersonal Disorders. PsyArxiv. https://doi.org/10.31234/osf.io/rck39

Zeeck, A., Schlegel, S., Giel, K. E., Junne, F., Kopp, C., Joos, A., Davis, C., \& Hartmann, A. (2017). Validation of the German Version of the Commitment to Exercise Scale. Psychopathology, 50(2), 146-156. https://doi.org/10.1159/000455929

Zipfel, S., Lowe, B., Reas, D. L., Deter, H. C., \& Herzog, W. (2000). Long-term prognosis in anorexia nervosa: lessons from a 21-year follow-up study. Lancet, 355(9205), 721-722. https://doi.org/10.1016/S0140-6736(99)05363-5 


\section{Tables}

Table 1. Sample Characteristics at Admission and Discharge from Inpatient Treatment.

\begin{tabular}{|c|c|c|c|c|c|c|c|c|}
\hline & \multicolumn{4}{|c|}{ Admission } & \multicolumn{4}{|c|}{ Discharge } \\
\hline & $n$ & $M$ & $S D$ & Range & $n$ & $M$ & $S D$ & Range \\
\hline Age (years) & 207 & 19.23 & 5.52 & $13-44$ & & & & \\
\hline Duration of illness (years) & 206 & 3.72 & 4.48 & $0.25-27$ & & & & \\
\hline Length of inpatient treatment (days) & & & & & 207 & 100.71 & 42.91 & $9-253$ \\
\hline BMI $\left(\mathrm{kg} / \mathrm{m}^{2}\right)$ & 207 & 15.17 & 1.92 & $11.1-21.99$ & 207 & 18.04 & 1.70 & $12.11-24.84$ \\
\hline BMI-SDS & 207 & -3.38 & 1.64 & $-7.85-0.46$ & 207 & -1.49 & 1.04 & $-5.22-1.26$ \\
\hline \multicolumn{9}{|l|}{ Questionnaire scores } \\
\hline Compulsivity (DAPP) & 202 & 3.61 & 0.71 & $1.53-5$ & & & & \\
\hline Disordered Eating (EDE-Q) & 183 & 4.30 & 1.14 & $1.09-6$ & 185 & 2.28 & 1.40 & $0-5.55$ \\
\hline Driven exercise (CES) & 206 & 2.93 & 0.68 & $1-4$ & 169 & 2.20 & 0.72 & $1-4$ \\
\hline \multicolumn{9}{|l|}{ Affect-regulatory difficulties } \\
\hline (DERS) & 202 & 3.19 & 0.76 & $1.36-4.75$ & 171 & 2.73 & 0.80 & $1.14-4.56$ \\
\hline \multicolumn{9}{|l|}{ Interpersonal sensitivity } \\
\hline (EDI-SI) & 188 & 3.72 & 0.87 & $1.88-6$ & 183 & 3.21 & 0.91 & $1.25-5.75$ \\
\hline
\end{tabular}

Note. $N=207, n$ varies due to missing values. BMI: Body mass index $\left(\mathrm{kg} / \mathrm{m}^{2}\right)$, BMI-SDS: ageand sex-standardized BMI, CES: Commitment to Exercise Scale used for assessing driven exercise, DAPP-Comp: Dimensional Assessment of Personality Pathology Compulsivity 
subscale, DERS: Difficulties in Emotion Regulation Scale global score, EDE-Q: Eating Disorder Examination questionnaire global score, EDI-SI: Eating Disorder Inventory-2 Social Insecurity subscale. For the sake of clarity, only admission and discharge scores were displayed (more details are available in Dittmer et al., 2020).

Table 2. Within-person and Between-person Correlations for Key Measures.

\begin{tabular}{llcccccc}
\hline \multicolumn{1}{c}{ Variable } & 1 & 2 & 3 & 4 & 5 & 6 \\
\hline 1 & BMI & -- &.- .60 &.- .53 & -.40 & $\mathbf{- . 3 8}$ & -- \\
2 & Disordered Eating (EDE-Q) & .10 & -- & $\mathbf{. 6 5}$ & $\mathbf{. 5 6}$ & $\mathbf{. 5 8}$ & - \\
3 & Driven exercise (CES) & -.01 & $\mathbf{. 5 6}$ & -- & $\mathbf{. 4 6}$ & $\mathbf{. 5 4}$ & - \\
4 & Interpersonal sensitivity (EDI-SI) & .01 & $\mathbf{. 5 1}$ & $\mathbf{. 2 7}$ & -- & $\mathbf{. 6 2}$ & - \\
5 & Affect-regulatory difficulties (DERS) & $<.01$ & $\mathbf{. 6 1}$ & $\mathbf{. 3 0}$ & $\mathbf{. 7 1}$ & -- & - \\
6 & Compulsivity (DAPP) & -.02 & $\mathbf{. 1 9}$ & $\mathbf{. 2 3}$ & $\mathbf{. 2 0}$ & $\mathbf{. 1 9}$ & - \\
7 & Age (in years) & .11 & .05 & .13 & .05 & .04 & $\mathbf{. 1 8}$ \\
\hline
\end{tabular}

Note. $N=207$ (between), $N=937-1035$ (within); Within-person correlations are displayed above the diagonal, between-person correlations are displayed below. BMI: Body Mass Index $\left(\mathrm{kg} / \mathrm{m}^{2}\right)$, CES: Commitment to Exercise Scale used for assessing driven exercise, DAPP-Comp: Dimensional Assessment of Personality Pathology Compulsivity subscale, DERS: Difficulties in Emotion Regulation Scale global score, EDE-Q: Eating Disorder Examination questionnaire global score, EDI-SI: Eating Disorder Inventory-2 Social Insecurity subscale. Missing values were excluded pairwise. Bold correlations indicate statistical significance at $p<.01$. 
Table 3. Key Standardized Coefficients from Multilevel Structural Equation Models Predicting Driven Exercise from Affect-regulatory Difficulties or

\section{Interpersonal Sensitivity and Moderation by Compulsivity.}

\begin{tabular}{|c|c|c|c|c|c|c|c|c|}
\hline & \multicolumn{4}{|c|}{ A: Affect Regulation } & \multicolumn{4}{|c|}{ B: Interpersonal Sensitivity } \\
\hline & \multicolumn{2}{|c|}{ Aim 1 Model } & \multicolumn{2}{|c|}{ Aim 2 Model } & \multicolumn{2}{|c|}{ Aim 1 Model } & \multicolumn{2}{|c|}{ Aim 2 Model } \\
\hline & $\beta$ & $\mathrm{CI}$ & $\beta$ & $\mathrm{CI}$ & $\beta$ & $\mathrm{CI}$ & $\beta$ & CI \\
\hline \multicolumn{9}{|l|}{ Within-person } \\
\hline Affect-regulatory difficulties $\rightarrow$ driven exercise & .37 & $.29-.44$ & .36 & $.28-.42$ & & & & \\
\hline Interpersonal sensitivity $\rightarrow$ driven exercise & & & & & .27 & $.18-.35$ & .24 & $.17-.33$ \\
\hline
\end{tabular}

\section{Between-person}

Affect-regulatory difficulties $\leftrightarrow$ driven exercise

$.28 \quad .12-.44 \quad .26 \quad .10-.41$

Compulsivity $\rightarrow$ affect-regulatory difficulties

$.04-.32$

Interpersonal sensitivity $\leftrightarrow$ driven exercise

$-\quad-$

.27

$.12-.43$

$.25 \quad .10-.39$

Compulsivity $\rightarrow$ interpersonal sensitivity

Compulsivity $\rightarrow$ driven exercise

\section{Cross-level moderation}

Compulsivity $\rightarrow$ random slope

$.02-.22-.28$

.47

$.24-.70$

Note. $N=207$ (between), $N=937$ (within); $\rightarrow$ indicates regression, $\leftrightarrow$ indicates covariation; model parameter estimates are standardized. $95 \%$ CIs of parameter estimates are displayed. Bolded values indicate the CI does not contain zero. In the Aim 2 model for affect-regulatory difficulties, a positive cross-level effect emerged for intervention allocation, which was included to control for treatment effects. This indicates that in the intervention group, the link between affect-regulatory difficulties and driven exercise 
was more pronounced. This effect was not observed in the Aim 2 model for interpersonal sensitivity. Within-person negative effects for assessment time point and current body weight were observed in all models. Positive between-person effects for age emerged in both aim 1 models and in the aim 2 model for interpersonal sensitivity. 


\section{Figures}

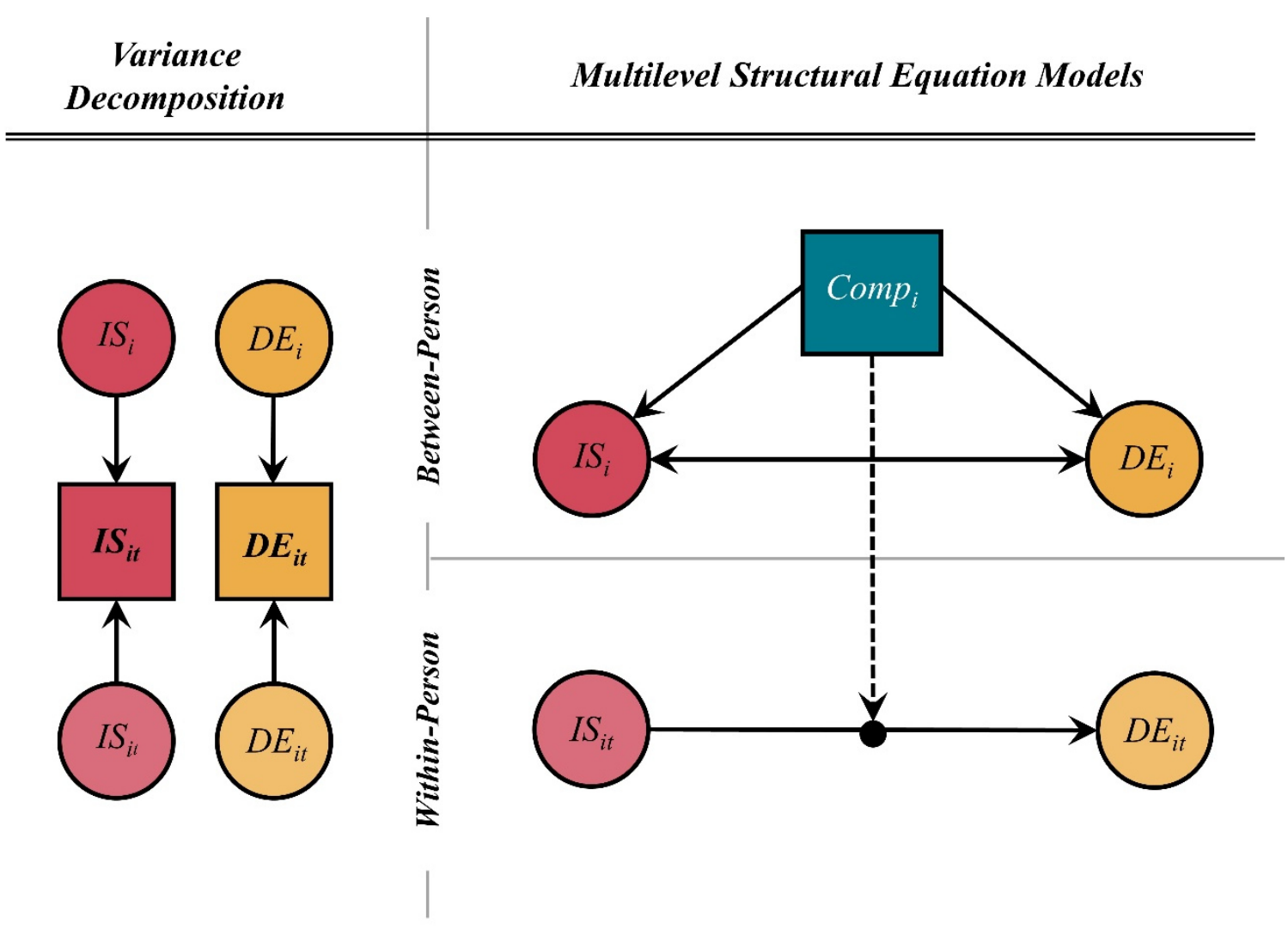

Figure 1. Schematic overview of estimated multilevel structural equation models. The panel on the left depicts the latent decomposition of observed longitudinally assessed variables into between- (subscript $i$ ) and within-person (subscript $i t$ ) variance. The right panel depicts the within-person (bottom) and between-person (top) portion of our focal model (Aim 2). Solid dots represent random slopes on within-person regression paths, dashed line represents crosslevel moderation. Parameters not reported in the tables (e.g., residual variances, covariates at both levels; age, RCT; time, BMI) are not depicted in diagrams, but respective Mplus code providing full models specification can be found online at: https://osf.io/ps53y/. DE: Driven exercise. IS: Interpersonal sensitivity. Comp: Dispositional compulsivity. 


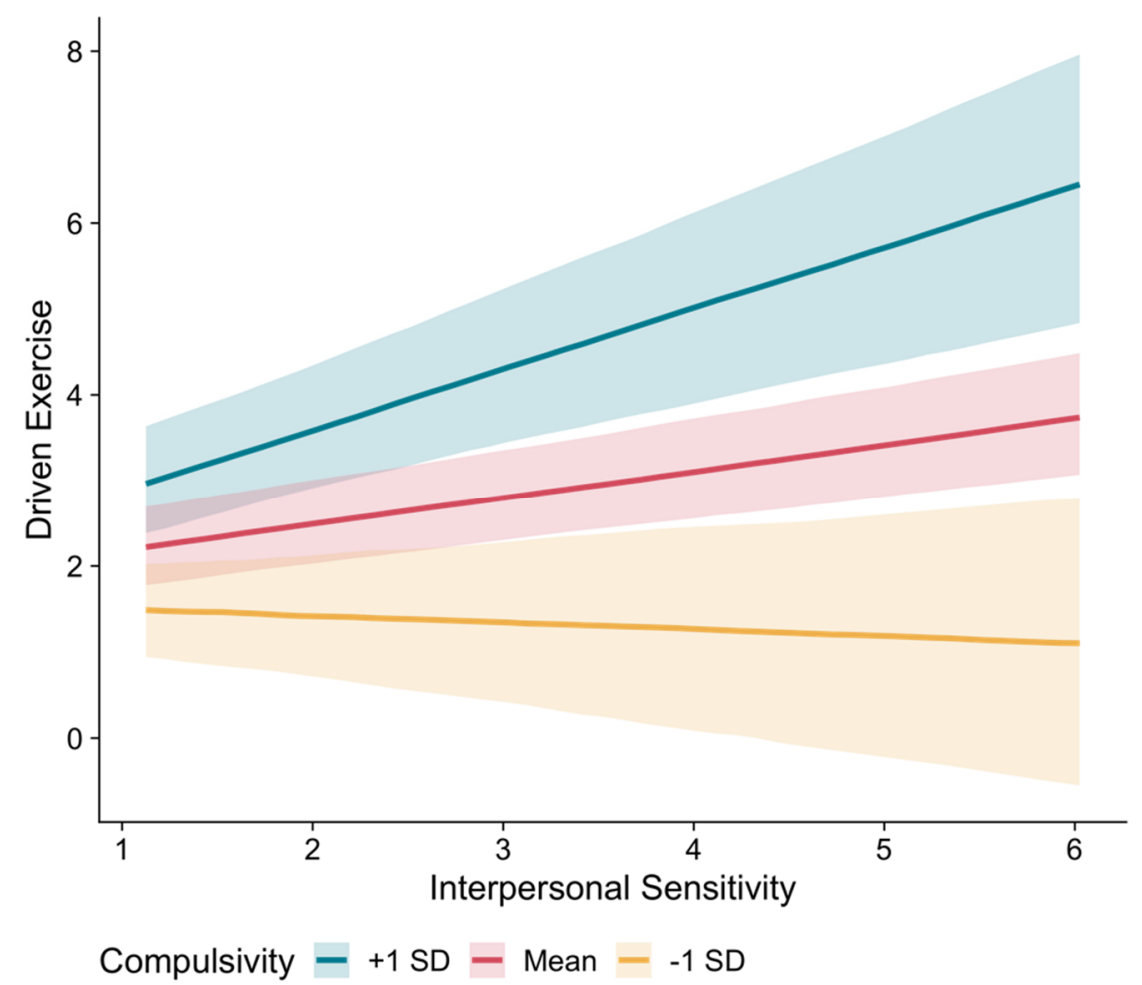

Figure 2. Distribution of random effects of interpersonal sensitivity on driven exercise as a function of dispositional compulsivity. Red line shows the average expected effect at mean levels of compulsivity, blue for $+1 S D$ above and yellow for $-1 S D$ below the mean. Shaded areas indicate $95 \%$ confidence intervals bands. Note that analyses were based on continuous compulsivity scores, that is, there was no categorization in compulsivity groups. This depiction only serves the purpose of visualizing the interaction effect between compulsivity and interpersonal sensitivity on driven exercise. 
Interpersonal, Affective and Compulsive Features of Driven Exercise in Anorexia Nervosa

\section{Supplementary Files}

Kolar, D. R. ${ }^{1, \dagger, *}$, Kaurin, A. ${ }^{2, \dagger}$, Meule, A. ${ }^{1,3}$, Schlegl, S. ${ }^{1,3}$, Dittmer, N. ${ }^{3}, \&$ Voderholzer, $\mathrm{U}^{1,3,4}$

${ }^{1}$ Department of Psychiatry, Ludwig-Maximilians-University Munich, Munich, Germany

${ }^{2}$ Faculty of Health/School of Psychology and Psychiatry, Witten/Herdecke University ${ }^{3}$ Schoen Clinic Roseneck, Prien am Chiemsee, Germany

${ }^{4}$ Department of Psychiatry and Psychotherapy, University Medical Center Freiburg, Freiburg, Germany

${ }^{\dagger}$ shared first authorship

*Corresponding author:

LMU University Hospital, Department of Psychiatry and Psychotherapy, Nussbaumstr. 7, 80336 Munich, Germany

Tel.: +49894400-52721

Fax.: +49 $894400-54572$

Email: David.kolar@med.uni-muenchen.de 
Supplementary file 1

Figure S1. Longitudinal Individual Differences in and Within-Person Variability of Key Measures at Assessments Across Treatment Groups.
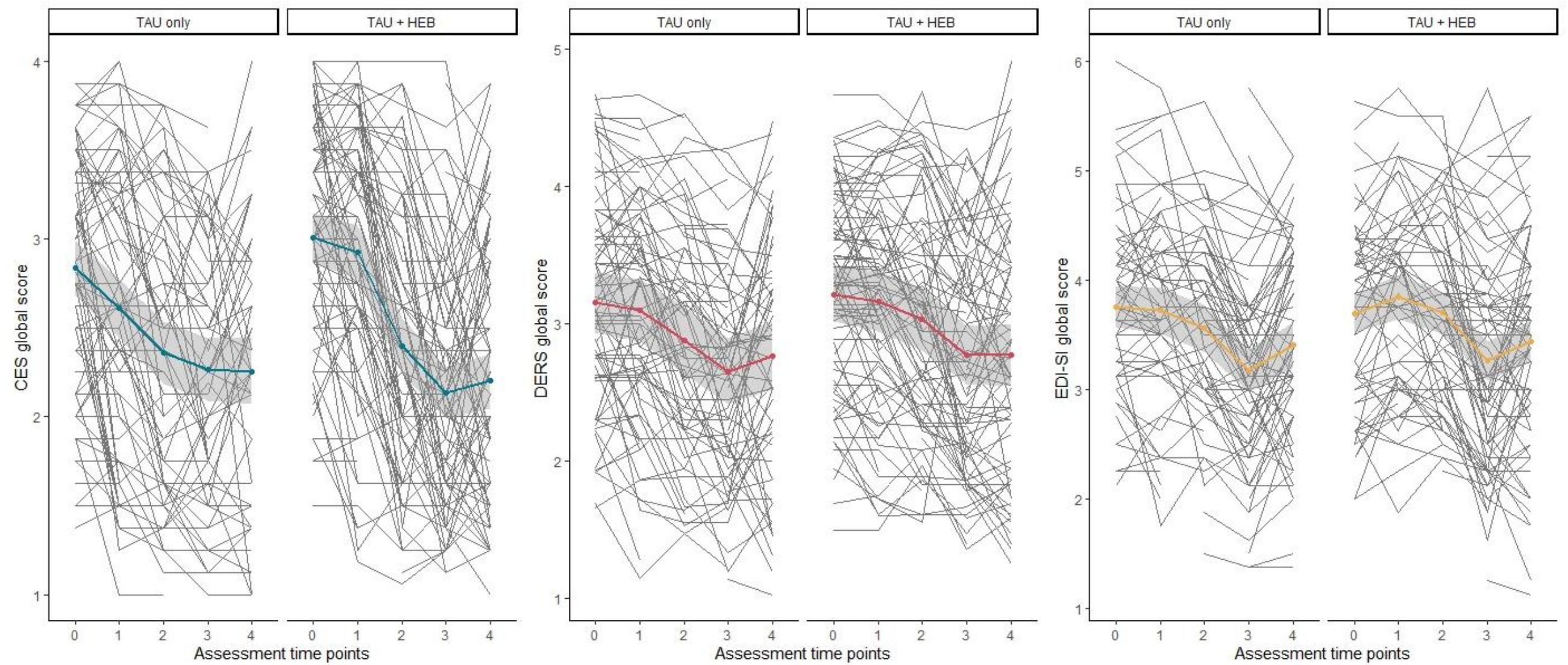

Note. CES: Commitment to Exercise Scale, DERS: Difficulties in Emotion Regulation Scale, EDI-SI: Eating Disorder Inventory-2 Social Insecurity subscale, TAU only: Treatment As Usual group, TAU + HEB: Treatment As Usual and additional Healthy Exercise Behavior group treatment. Assessment time points: Self-report measures were assessed at five timepoints: admission (T0; within the first week after admission), pre-intervention (T1; within three days before the start of the healthy exercise behavior group intervention), post-intervention (T2; within three days after the last intervention session, roughly four weeks after T1), discharge (T3, within one week before discharge) and six months follow-up (T4). Colored lines represent group mean scores with 95\% CI bands; grey lines are individual mean scores. 


\section{Supplementary file 3}

Table S2. Assessment Schedule.

\begin{tabular}{|c|c|c|c|c|c|}
\hline \multirow[b]{2}{*}{ Instrument } & \multicolumn{5}{|c|}{ Assessment time point } \\
\hline & $\begin{array}{c}\text { T0 } \\
\text { Admission }\end{array}$ & $\begin{array}{c}\mathrm{T} 1 \\
\text { Pre-Intervention }\end{array}$ & $\begin{array}{c}\mathrm{T} 2 \\
\text { Post-Intervention }\end{array}$ & $\begin{array}{c}\text { T3 } \\
\text { Discharge }\end{array}$ & $\begin{array}{c}\mathrm{T} 4 \\
\text { Follow-Up }\end{array}$ \\
\hline SIAB-EX & $\mathbf{x}$ & & & & \\
\hline Diagnostic Compulsive Exercise Interview & $\mathbf{x}$ & & & & \\
\hline BMI $\left(\mathrm{kg} / \mathrm{m}^{2}\right)$ & $\mathbf{x}$ & & & & \\
\hline DAPP-Compulsivity & $\mathbf{x}$ & & & & \\
\hline Weight & $\mathbf{x}$ & $\mathbf{x}$ & $\mathbf{x}$ & $\mathbf{x}$ & $\mathbf{x}$ \\
\hline CES & $\mathbf{x}$ & $\mathbf{x}$ & $\mathbf{x}$ & $\mathbf{x}$ & $\mathbf{x}$ \\
\hline EDE-Q & $\mathbf{x}$ & $\mathbf{x}$ & $\mathbf{x}$ & $\mathbf{x}$ & $\mathbf{x}$ \\
\hline DERS & $\mathbf{x}$ & $\mathbf{x}$ & $\mathbf{x}$ & $\mathbf{x}$ & $\mathbf{x}$ \\
\hline EDI-SI & $\mathbf{x}$ & $\mathbf{x}$ & $\mathbf{x}$ & $\mathbf{x}$ & $\mathbf{x}$ \\
\hline
\end{tabular}

Note: Assessment time points: T0 (within the first week after admission), T1 (within three days before the start of the healthy exercise behavior group intervention), T2 (within three days after the last intervention session, roughly four weeks after T1), T3 (within one week before discharge) and T4 (six months after T3). BMI: Body Mass Index in $\mathrm{kg} / \mathrm{m}^{2}$; CES: Commitment to Exercise Scale; DAPP-Compulsivity: Dimensional Assessment of Personality Pathology - Compulsivity Subscale; DERS:

Difficulties in Emotion Regulation Scale; Diagnostic Compulsive Exercise Interview: Stuctured interview to classify patients as compulsive exercisers; EDE-Q: Eating Disorder Examination Questionnaire; EDI-SI: Eating Disorder Inventory-2 - Social Insecurity Subscale. SIAB-EX: Structured Inventory for Anorexic and Bulimic Eating Disorders - Expert Interview. 
Supplementary file 3

Table S3. Full Standardized Coefficients from MSEMs Predicting Compulsive Exercise from Affect-regulatory Difficulties or Interpersonal Sensitivity and Moderation by Compulsivity.

\begin{tabular}{|c|c|c|c|c|c|c|c|c|}
\hline & \multicolumn{4}{|c|}{ A: Affect Regulation Models } & \multicolumn{4}{|c|}{ B: Interpersonal Sensitivity Models } \\
\hline & \multicolumn{2}{|c|}{ Aim 1 Model } & \multicolumn{2}{|c|}{ Aim 2 Model } & \multicolumn{2}{|c|}{ Aim 1 Model } & \multicolumn{2}{|c|}{ Aim 2 Model } \\
\hline & $\beta$ & $C I$ & $\beta$ & $C I$ & $\beta$ & $C I$ & $\beta$ & $C I$ \\
\hline \multicolumn{9}{|l|}{ Within-person } \\
\hline ARD/IS $\rightarrow$ driven exercise & .37 & $.29-.44$ & .36 & $.28-.42$ & .27 & $.18-.35$ & .24 & $.17-.33$ \\
\hline Assessment time point $\rightarrow$ driven exercise & -.33 & $-.40--.25$ & -.30 & $-.36--.24$ & -.35 & $-.44--.27$ & -.36 & $-.43--.28$ \\
\hline Weight $\rightarrow$ driven exercise & -.07 & $-.11--.01$ & -.10 & $-.12--.08$ & -.11 & $-.15--.01$ & -.10 & $-.15--.01$ \\
\hline \multicolumn{9}{|l|}{ Between-person } \\
\hline Treatment group $\rightarrow$ driven exercise & -.02 & $-.18-.13$ & -.02 & $-.16-.13$ & -.01 & $-.16-.14$ & -.02 & $-.16-.13$ \\
\hline Treatment group $\rightarrow$ ARD/IS & .03 & $-.10-.17$ & .03 & $-.12-.17$ & .03 & $-.11-.17$ & .02 & $-.13-.17$ \\
\hline Age $\rightarrow$ driven exercise & .19 & $.03-.33$ & .14 & $-.01-.29$ & .19 & $.04-.32$ & .16 & $.01-.31$ \\
\hline Age $\rightarrow$ ARD/IS & .04 & $-.13-.19$ & .01 & $-.14-.15$ & .05 & $-.12-.20$ & .01 & $-.13-.15$ \\
\hline ARD/IS $\leftrightarrow$ driven exercise & .28 & $.12-.44$ & .26 & $.10-.41$ & .27 & $.12-.43$ & .25 & $.10-.39$ \\
\hline Compulsivity $\rightarrow$ ARD/IS & - & - & .19 & $.04-.32$ & - & - & .21 & $.06-.36$ \\
\hline Compulsivity $\rightarrow$ driven exercise & - & - & .22 & $.07-.36$ & - & - & .21 & $.06-.36$ \\
\hline \multicolumn{9}{|l|}{ Cross-level } \\
\hline Driven exercise $\leftrightarrow$ random slope & -.01 & $-.29-.26$ & .01 & $-.29-.29$ & .22 & $-.09-.54$ & .14 & $-.23-.44$ \\
\hline ARD/IS $\leftrightarrow$ random slope & -.20 & $-.49-.10$ & -.19 & $-.46-.13$ & -.14 & $-.51-.21$ & -.32 & $-.72-.17$ \\
\hline Treatment group $\rightarrow$ random slope & .25 & $-.01-.48$ & .24 & $.01-.47$ & .15 & $-.13-.40$ & .17 & $-.14-.44$ \\
\hline Age $\rightarrow$ random slope & .02 & $-.18-.25$ & .01 & $-.21-.24$ & -.23 & $-.52-.17$ & -.26 & $-.47-.02$ \\
\hline $\begin{array}{l}\text { Compulsivity } \rightarrow \text { random slope } \\
\text { (ARD/IS } \rightarrow \text { driven exercise) }\end{array}$ & - & - & .02 & $-.22-.28$ & - & - & .47 & $.24-.70$ \\
\hline
\end{tabular}

Note: $N=207$ (between), $N=1035$ (within); ARD: affect-regulatory difficulties, IS: interpersonal sensitivity. The

Difficulties in Emotion Regulation Scale global score was used to measure affect-regulatory difficulties, and the Eating Disorders Inventory-2 Social Insecurity subscale score to measure interpersonal sensitivity; $\rightarrow$ indicates regression, $\leftrightarrow$ indicates covariation; model parameter estimates are standardized. $95 \%$ credibility intervals of parameter estimates are displayed. Bolded values indicate the credibility interval does not contain zero. Values $<.01$ are rounded to .01 . 
Supplementary file 4

Table S4. Full Standardized Coefficients from MSEMs Predicting Disordered Eating from Affect-regulatory Difficulties or Interpersonal Sensitivity and Moderation by Compulsivity.

\begin{tabular}{|c|c|c|c|c|c|c|c|c|}
\hline & \multicolumn{4}{|c|}{ A: Affect Regulation Models } & \multicolumn{4}{|c|}{ B: Interpersonal Sensitivity Models } \\
\hline & \multicolumn{2}{|c|}{ Aim 1 Model } & \multicolumn{2}{|c|}{ Aim 2 Model } & \multicolumn{2}{|c|}{ Aim 1 Model } & \multicolumn{2}{|c|}{ Aim 2 Model } \\
\hline & $\beta$ & $C I$ & $\beta$ & $C I$ & $\beta$ & $C I$ & $\beta$ & $C I$ \\
\hline \multicolumn{9}{|l|}{ Within-person } \\
\hline ARD/IS $\rightarrow$ disordered eating & .45 & $.38-.51$ & .44 & $.35-.51$ & .41 & $.32-.48$ & .40 & $.33-.47$ \\
\hline Assessment time point $\rightarrow$ disordered eating & -.32 & $-.39--.25$ &.- .33 & $-.40--.26$ & -.34 & $-.43--.27$ &.- .36 & $-.42--.30$ \\
\hline Weight $\rightarrow$ disordered eating & -.11 & $-.17--.05$ & -.09 & $-.14--.04$ & -.08 & $-.15--.01$ & -.07 & $-.13--.03$ \\
\hline \multicolumn{9}{|l|}{ Between-person } \\
\hline Treatment group $\rightarrow$ disordered eating & .05 & $-.11-.20$ & .03 & $-.12-.18$ & .05 & $-.11-.19$ & .05 & $-.11-.20$ \\
\hline Treatment group $\rightarrow$ ARD/IS & .04 & $-.11-.19$ & .02 & $-.12-.17$ & .03 & $-.12-.17$ & .02 & $-.12-.17$ \\
\hline Age $\rightarrow$ disordered eating & .11 & $-.05-.26$ & .08 & $-.07-.22$ & .12 & $-.03-.27$ & .07 & $-.08-.21$ \\
\hline Age $\rightarrow$ ARD/IS & .04 & $-.11-.19$ & .01 & $-.14-.15$ & .05 & $-.10-.20$ & .01 & $-.13-.16$ \\
\hline ARD/IS $\leftrightarrow$ disordered eating & .65 & $.53-.74$ & .65 & $.53-.74$ & .52 & $.39-.63$ & .51 & $.38-.63$ \\
\hline Compulsivity $\rightarrow$ ARD/IS & - & - & .18 & $.03-.33$ & - & - & .20 & $.05-.33$ \\
\hline Compulsivity $\rightarrow$ disordered eating & - & - & .18 & $.03-.33$ & - & - & .18 & $.02-.33$ \\
\hline \multicolumn{9}{|l|}{ Cross-level } \\
\hline Disordered eating $\leftrightarrow$ random slope & -.27 & $-.61-.05$ & -.32 & $-.68-.03$ & -.49 & $-.69--.13$ & -.59 & $-.84--.26$ \\
\hline ARD/IS $\leftrightarrow$ random slope & -.57 & $-.88--.18$ & -.52 & $-.84--.18$ & -.59 & $-.83--.26$ & -.65 & $-.92--.27$ \\
\hline Treatment group $\rightarrow$ random slope & -.14 & $-.50-.19$ & -.11 & $-.38-.17$ & -.23 & $-.53-.13$ & -.19 & $-.49-.11$ \\
\hline Age $\rightarrow$ random slope & -.17 & $-.50-.16$ & -.12 & $-.40-.16$ & -.04 & $-.34-.29$ & .01 & $-.30-.37$ \\
\hline $\begin{array}{l}\text { Compulsivity } \rightarrow \text { random slope } \\
(\text { ARD/IS } \rightarrow \text { disordered eating) }\end{array}$ & - & - & .04 & $-.27-.35$ & - & - & .08 & $-.25-.47$ \\
\hline
\end{tabular}

Note: $N=207$ (between), $N=1035$ (within); ARD: affect-regulatory difficulties, IS: interpersonal sensitivity. The

Difficulties in Emotion Regulation Scale global score was used to measure affect-regulatory difficulties, and the Eating Disorders Inventory-2 Social Insecurity subscale score to measure interpersonal sensitivity; $\rightarrow$ indicates regression, $\leftrightarrow$ indicates covariation; model parameter estimates are standardized. $95 \%$ credibility intervals of parameter estimates are displayed. Bolded values indicate the credibility interval does not contain zero. Values $<.01$ are rounded to .01. 